\title{
5-Aminolevulinic acid induced apoptosis via oxidative stress in normal gastric epithelial cells
}

\author{
Hiromu Ito, ${ }^{1}$ Hiromi Kurokawa, ${ }^{2}$ Hideo Suzuki, ${ }^{2}$ Hiroko P. Indo, ${ }^{1}$ Hideyuki J. Majima ${ }^{1}$ and Hirofumi Matsui ${ }^{2, *}$ \\ 'Graduate School of Medical and Dental Sciences, Kagoshima University, 8-35-1 Sakuragaoka, Kagoshima, Kagoshima 890-8544, Japan \\ ${ }^{2}$ Department of Gastroenterology, Faculty of Medicine, University of Tsukuba, 1-1-1 Tennoh-dai, Tsukuba, Ibaraki 305-8575, Japan
}

(Received 10 April, 2018; Accepted 4 January, 2019; Published online 9 August, 2019)

5-Aminolevulinic acid, a precursor of heme, is utilized in a variety of applications including cancer treatment, surgery, and plant nutrition. However, 5-aminolevulinic acid itself induces oxidative stress and subsequent lipid peroxidation. Reactive oxygen species are factors in oxidative stress, not only causing cellular injury but also inducing several signal transduction pathways. Especially in cancer cells, a significant amount of signalling activation and subsequent activation of protein is caused by the enhancement of reactive oxygen species production. Reactive oxygen species levels in normal cells are low and an oxidative condition is harmful; hence, administration of 5-aminolevulinic acid to normal cells may induce oxidative stress, resulting in cell death. In this study, we investigated the effect of 5 -aminolevulinic acid on normal and cancer cells with regard to oxidative stress. We used the rat normal gastric cell line RGM and its cancer-like mutant cell line RGK. 5-Aminolevulinic acid treatment of RGM cells enhanced reactive oxygen species generation and induced apoptosis associated with p53, whereas RGK cells were unaffected. In addition, RGM cell viability was recovered by application of $\mathrm{N}$-acetyl-L-cysteine or p53 inhibitor. These results suggest that 5 -aminolevulinic acid causes oxidative stress in normal gastric cells and induces apoptosis via the p53-dependent pathway.

Key Words: 5-aminolevulinic acid, reactive oxygen species, gastric epithelial cell, apoptosis

$\mathrm{H}$ eme is an essential substance for many kinds of animals to metabolize oxygen and generate energy. 5-Aminolevulinic acid (5-ALA) is a precursor of heme and has some positive effects on health, such as promoting hair growth and boosting the immune system. ${ }^{(1-3)} 5$-ALA is also utilized in photodynamic therapy (PDT). ALA-PDT is a combination therapy utilizing appropriate irradiation and a photosensitizer (protoporphyrin IX: PpIX) that is produced from 5-ALA. ${ }^{(4)}$ In recent years, this photochemical property has been used to diagnose brain tumours and determine the area of resection. ${ }^{(5)} 5$-ALA is also an important nutrient for plants. It is a basic ingredient of chlorophyll where is the photosynthetic reaction centre and accelerates plant growth. ${ }^{(6)}$ In addition, 5-ALA improves salt and cold resistance in plants. ${ }^{(7,8)}$ However, at high concentrations, 5-ALA causes photosensitivity and could act as an herbicide. ${ }^{(6,9)}$ It has been reported that 5-ALA induces formation of reactive oxygen species (ROS) that cause lipid peroxidation, particularly cardiolipin, a major phospholipid in the mitochondrial inner membrane. ${ }^{(10-12)}$

ROS have wide influence over organisms, affecting signalling, transcription factor activation, and the immune system. ${ }^{(1)}$ Overproduction of ROS results in oxidative stress and is associated with various intractable disease like cancer, Alzheimer's disease, and inflammatory bowel disease. ${ }^{(14-16)}$ ROS can be generated in the endoplasmic reticulum and through the action of NADPH oxidase in the cell membrane. ${ }^{(17,18)}$ However, mitochondria are the primary source of ROS because they consume oxygen in the electron transport chain (ETC), which produces ROS as a byproduct of energy production. ${ }^{(19,20)}$ Mitochondrial DNA (mtDNA) mutations can decrease the efficiency of ETC and enhance the production of ROS.(21) In general, larger amounts of ROS are produced in cancer cells than in normal cells because of mtDNA mutations. ${ }^{(22,23)}$ Over-production of ROS activates signalling pathways that induce protein expression. ROS derived from mitochondria have been implicated in metastasis and cancer invasiveness. ${ }^{(24,25)}$ Thus, ROS play an important role in maintenance of morphology of cancer cells and are essential factors for survival of cancer. On the other hand, oxidative stress is harmful to normal cells. ${ }^{(26)}$ Taken together, ROS produced by administration of 5-ALA may both elevate cancer activity and damage normal cells. Especially in ALA-PDT, normal tissue is also exposed by 5-ALA and to examine the effect is important for patients because the effect of photosensitizers for normal tissue which is not irradiated has not been discussed. In this study, we examined and compared the effect of 5-ALA on ROS production in normal and cancerous gastric cells. The rat normal gastric epithelial cell line, RGM and cancer-like cell line, RGK were used for comparison. RGM is derived from gastric mucosa of Wistar rats, and RGK is a chemically mutated strain of RGM. ${ }^{(27,28)} \mathrm{We}$ used 1-methyl-3-nitro-1-nitrosoguanidine (MNNG) to transform RGM to RGK. Since these cell lines have the same genetic background, they are suitable for comparing the behaviour of normal and cancer cells.

\section{Materials and Methods}

Cell culture. RGM, a normal gastric epithelial cell line derived from rat, and RGK, a cancer-like mutant cell line of RGM, were established previously. ${ }^{(27,28)}$ RGM and RGK cells were cultured in Dulbecco's modified Eagle medium/Nutrient Mixture F-12 (DMEM/F12) with (Thermo Fisher Scientific, Waltham, MA) and without (Sigma-Aldrich Co. LLC., St. Louis, MO) Lglutamine, respectively. Each medium was supplemented with $10 \%$ foetal bovine serum (Equitech-Bio Inc., Kerrville, TX) and $1 \%$ penicillin/streptomycin (Thermo Fisher Scientific). Cells were maintained at $37^{\circ} \mathrm{C}$ in moist air with $5 \% \mathrm{CO}_{2}$. All cell incubation with 5-ALA was performed in the dark condition.

Cell viability test. Cell viability, which means cellular proliferation ability, after 5-ALA exposure was calculated using the Cell Counting Kit-8 (CCK-8) colorimetric assay (DOJINDO LABORATORIES, Kumamoto, Japan), which is an alternative method of MTT assay, according to the manufacturer's protocol.

*To whom correspondence should be addressed. E-mail: hmatsui@md.tsukuba.ac.jp 
Briefly, RGM and RGK cells were seeded on a 96-well cell culture plate at a density of 5,000 cells/well and incubated overnight at $37^{\circ} \mathrm{C}$ in $5 \% \mathrm{CO}_{2}$. The medium of each well was replaced with fresh media containing $1,5,10,50,100,500$ and $1,000 \mu \mathrm{M} 5$ ALA (Cosmo Bio Co., Ltd., Tokyo, Japan). 5-ALA is cleared from tissue and body in $48 \mathrm{~h} .{ }^{(29)}$ Thus, cells were incubated for $48 \mathrm{~h}$ before the 5-ALA containing medium was removed and cells were washed with PBS twice. Cells were transferred to fresh medium containing $10 \%(\mathrm{v} / \mathrm{v})$ CCK-8 solution and additionally incubated to develop colour. The absorbance of each well at $450 \mathrm{~nm}$ was measured with a DTX880 multi-mode micro plate reader (Beckman Coulter Inc., Brea, CA).

Annexin V assay. Flow cytometric analysis with annexin V was used to determine the cellular state after 5-ALA exposure. In apoptotic phase, phosphatidylserine (PS), a constituent of the inner cell membrane, is exposed to the cell exterior. Annexin V binds to PS in a calcium dependent manner. This characteristic of annexin $\mathrm{V}$ is often utilized to study cellular apoptosis. We used Muse Annexin V \& Dead Cell Kit (EMD Millipore) according to the manufacturer's instructions to determine the ratio of programmed cell death after 5-ALA treatment. RGM and RGK cells cultured in $10 \mathrm{~cm}$ dishes were exposed to $50 \mu \mathrm{M} 5$-ALA and incubated for $48 \mathrm{~h}$ at $37^{\circ} \mathrm{C}$. Cells were washed with PBS and harvested with trypsin. Cell concentration was adjusted to $3 \times 10^{5}$ cells $/ \mathrm{ml}$, and $100 \mu \mathrm{l}$ of cell suspension was mixed thoroughly with an equal volume of Muse Annexin V \& Dead Cell. The cells were incubated for $20 \mathrm{~min}$ in the dark at $25^{\circ} \mathrm{C}$. After incubation, the cell suspension was transferred to a $1.5 \mathrm{ml}$ tube and analysed by the Muse Cell Analyzer in Annexin V \& Dead Cell mode.

Caspase assay. Flow cytometric analysis focused on caspase was used to examine apoptosis by a separate method. Caspases are cysteine-aspartic proteases that play essential roles in programmed cell death via complex signal cascades. Their expression is an indicator of apoptosis. ${ }^{(30)} \mathrm{We}$ used the Muse Caspase-3/7 Kit (EMD Millipore) to distinguish caspase-3 and caspase-7 positive from negative cells to produce the apoptotic ratio after 5-ALA treatment. Briefly, RGM and RGK cells cultured in $10 \mathrm{~cm}$ dishes were treated with $50 \mu \mathrm{M} 5$-ALA for $48 \mathrm{~h}$ in $5 \% \mathrm{CO}_{2}$ at $37^{\circ} \mathrm{C}$. Cells were washed with PBS and collected with trypsin. Cell concentration was adjusted to $3 \times 10^{5}$ cells $/ \mathrm{ml}$ in $50 \mu$ l of $1 \mathrm{X}$ Assay Buffer BA from the kit. Next, $5 \mu \mathrm{l}$ of Muse Caspase-3/7 Reagent working solution was added to the cells and incubated in $5 \% \mathrm{CO}_{2}$ at $37^{\circ} \mathrm{C}$ for $30 \mathrm{~min}$. After incubation, $150 \mu \mathrm{l}$ of Muse Caspase 7-AAD working solution was added and mixed thoroughly. The suspension was incubated with light shielding at $25^{\circ} \mathrm{C}$ for $5 \mathrm{~min}$. Cells were analysed by the Muse Cell Analyzer in Caspase-3/7 assay mode.

Western blotting. The levels of caspase-9 and p53 after 5ALA treatment were investigated by western blotting using a previously published method. ${ }^{(31)}$ RGM and RGK cells post-5-ALA treatment were collected and treated with NuPAGE LDS Sample Buffer (Thermo Fisher Scientific). The cell samples were heated at $95^{\circ} \mathrm{C}$ for $5 \mathrm{~min}$ and $10 \mu \mathrm{l}$ was added to the wells of NuPAGE $12 \%$ Bis-Tris Protein Gels (Thermo Fisher Scientific). Proteins were separated by polyacrylamide gel electrophoresis (PAGE) and transferred to polyvinylidene difluoride (PVDF) membrane (EMD Millipore). The membrane was washed with PBS containing $0.1 \%$ Tween 20 (PBS-T) for 5 min and treated with PVDF Blocking Reagent from Can Get Signal (TOYOBO Co., Ltd., Osaka, Japan) for $60 \mathrm{~min}$ at $25^{\circ} \mathrm{C}$. After blocking, the membrane was washed three times with PBS-T for 10 min followed by overnight incubation with primary antibodies diluted $1: 1,000$ in Can Get Signal Immunoreaction Enhancer Solution 1: caspase-9 (Cell Signaling Technology, Danvers, MA), phospho-p53 (Cell Signaling Technology) or p53 (Cell Signaling Technology). After primary antibody incubation, the membrane was washed three times with PBS-T and incubated with horseradish peroxidase-conjugated anti-rabbit IgG antibody (Cell Signaling Technology) diluted $1: 1,000$ in Can Get Signal Immunoreaction Enhancer Solution 2 for $2 \mathrm{~h}$. The secondary antibody solution was removed and the membrane was washed three times with PBS-T. The membrane was immersed in Lumina forte western HRP substrate (EMD Millipore) and luminescence was detected with a LAS4000 instrument (GE Health Care Japan, Tokyo, Japan). As a loading control, $\beta$-actin was detected with $\beta$-actin primary antibody (Cell Signaling Technology).

ROS detection by electron spin resonance. ROS generation in living cells after 5-ALA treatment was examined by electron spin resonance (ESR) according to a previously published method. ${ }^{(25)}$ RGM and RGK cells were cultured on glass cover slides $(49 \times 5 \times 0.2 \mathrm{~mm})$ and treated with $50 \mu \mathrm{M} \mathrm{5}$-ALA for $48 \mathrm{~h}$. A cell-bearing slide was set in tissue glass and $100 \mu \mathrm{l}$ of respiratory buffer [ $5 \mathrm{mM}$ succinate, $5 \mathrm{mM}$ malate, $5 \mathrm{mM}$ glutamate, $5 \mathrm{mM}$ nicotinamide adenine dinucleotide (NADH), and 5\% 5,5-dimethyl1 -pyrroline- $N$-oxide (DMPO)] was added. The tissue glass was inserted in an ESR device, a JEOL-TE X-band spectrometer (JEOL Ltd., Tokyo, Japan). Spectra were obtained under $10 \mathrm{~mW}$ incident microwave power, $9.42 \mathrm{GHz}$ frequency, and $0.1 \mathrm{mT}$ field modulation amplitude.

$\boldsymbol{N}$-acetyl-L-cysteine treatment. RGM cells were exposed to $\mathrm{N}$-acetyl-L-cysteine (NAC), which is an antioxidant, and 5-ALA, followed by cell viability measurement using CCK-8 according to the method described in section 2.2 , supplementing with $50 \mu \mathrm{M}$ 5-ALA and increasing concentrations $(0,1,5,10,50,100$ and $500 \mu \mathrm{M}$ ) of NAC (Wako Pure Chemical Industries, Ltd., Osaka, Japan).

p53 inhibitor treatment. Cells were treated with p53 inhibitor and 5-ALA, then cell viability was measured to examine induction of the apoptotic pathway by 5-ALA. RGM cells were cultured in 96-well plates at 5,000 cells/well and incubated overnight. The medium was removed and replaced with fresh medium containing increasing concentrations $(0,1,2,5,10,20,50$ and $100 \mu \mathrm{M})$ of an inhibitor of p53-dependent apoptosis and transcription, Cyclic Pifithrin- $\alpha$ hydrobromide (Tokyo Chemical Industry Co., Ltd., Tokyo, Japan). After $24 \mathrm{~h}$ incubation, the p53 inhibitor was removed and cells were washed with PBS. Cells were additionally incubated in medium containing $50 \mu \mathrm{M} 5$-ALA for $48 \mathrm{~h}$ and the CCK-8 assay was performed as described in section 2.2.

Statistical analysis. Statistical analysis was carried out using SPSS statistics 21 software (IBM Corporation, NY). Tukey's test was used to compare more than two data sets and Student's $t$ test was used for two data sets. $P<0.05$ and $p<0.01$ were considered as statistically significant differences. All data are represented as mean \pm SD.

\section{Results}

Cytotoxic effect of 5-ALA on gastric normal and cancer cells. Rat gastric normal cell line RGM and cancer cell line RGK were treated with 5-ALA for $48 \mathrm{~h}$ and cytotoxicity was calculated by the CCK-8 method. RGM cells showed a gradual decrease in viability dependent on the 5-ALA dose, up to $50 \mu \mathrm{M}$, and the viability gradually recovered (Fig. 1). On the other hand, RGK cancer cells proliferated in a 5-ALA-dose dependent manner up to $5 \mu \mathrm{M}$, and their numbers decreased from $5 \mu \mathrm{M}$ to $100 \mu \mathrm{M}$. After $1,5,10,50,500$ and $1,000 \mu \mathrm{M} 5$-ALA treatment; viability of RGM cells decreased significantly compared to that of RGK cells. These results indicate that 5-ALA is toxic to normal gastric cells, especially at $50 \mu \mathrm{M}$, but promotes the growth of cancer cells at concentrations up to $50 \mu \mathrm{M}$ (Fig. 1 and Supplemental Fig. $1 *$ ). As described above, 5-ALA also has characteristics to promote cellular growth such as hair and plants. Cancer cells may have higher sensitivity to 5-ALA.

Apoptosis measurement by annexin V and caspase-3/7 assay. Cellular apoptosis was studied using Muse Annexin V \& Dead Cell Kit after exposure to $50 \mu \mathrm{M}$ 5-ALA for 48 h. Fig. 2 shows the clear change of histogram in RGM cells after 5-ALA 


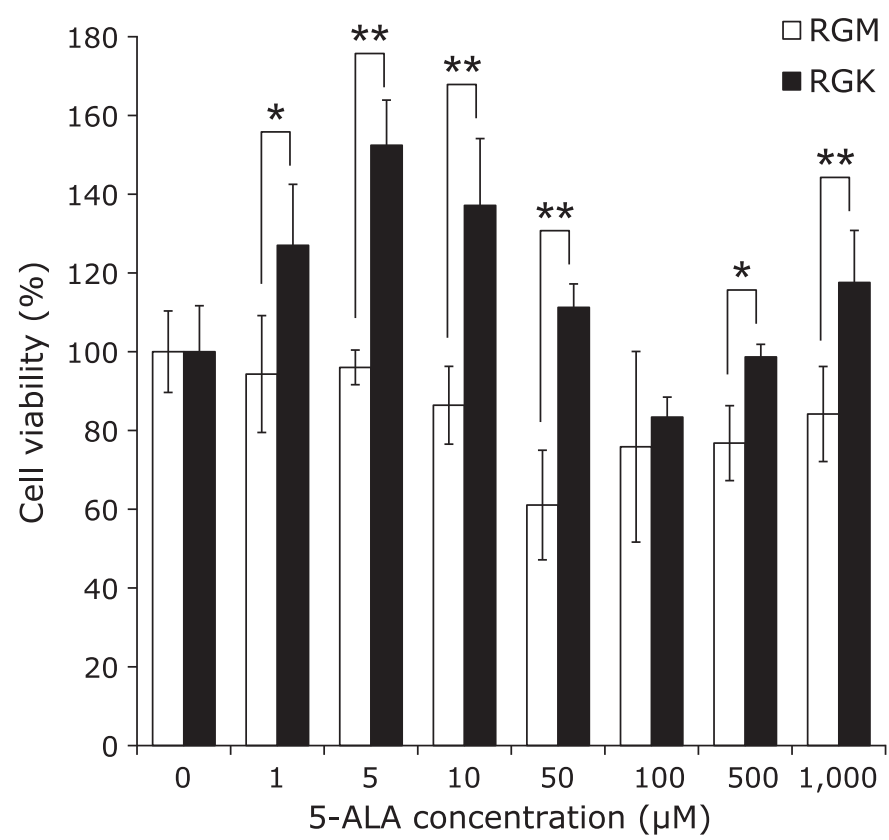

Fig. 1. Cell viability of RGM normal gastric epithelial cells and RGK cancer-like gastric cells after treatment with 5-ALA for $48 \mathrm{~h}$, measured by the CCK-8 colorimetric method. Viability of RGK cells increased with treatment of 5-ALA whereas viability of RGM cells decreased in a dosedependent manner up to $50 \mu \mathrm{M}$. Statistical significance was tested by Student's $t$ test. $n=4$, Error bar; SD. ${ }^{*} p<0.05,{ }^{*} p<0.01$.

treatment. The late apoptotic or dead cell ratio of RGM cells increased significantly after 5-ALA treatment. In RGK cells, the ratio did not change much and the early apoptotic ratio increased slightly. The cellular apoptotic ratio was also measured and calculated by a caspase-3/7 assay. Figure 3 shows most RGM cells without treatment were living and the apoptotic/dead ratio was $3.32 \%$. Exposure to $50 \mu \mathrm{M} 5$-ALA increased the ratio to $22.18 \%$. On the other hand, the apoptotic/dead ratio in RGK cells was not changed by 5-ALA (from 3.96 to $3.42 \%$ ) and over $90 \%$ of cells were living. These results indicate that $50 \mu \mathrm{M}$ 5-ALA easily influences the viability of gastric normal cells compared to cancer cells and induces an apoptotic state. In comparison between Fig. 2 and 3 , there was a little difference in the value of living and apoptotic ratio. These results may be from the difference of way to measure. However, ALA treatment obviously increased apoptotic indication in RGM cells in both results. Thus, 5-ALA induces apoptotic signal transduction in RGM normal cells easier than RGK cancer cells.

Protein expression analysis by western blotting. Caspase proteins are involved in many signalling cascades and are deeply involved with apoptosis. One of the caspase proteins, caspase-9, especially takes part in the mitochondrial apoptotic pathway via oxidative stress. ${ }^{(32,33)}$ Normal p53 protein also induces cell death in response to high oxidative stress. ${ }^{(34)} \mathrm{We}$ examined the changes in expression levels of these proteins after 5-ALA treatment to clarify the signalling pathway and mechanism of apoptosis derived from 5-ALA treatment. Figure 4A shows that the level of cleaved caspase-9 increased after 5-ALA treatment in RGM cells. In RGK cells, the caspase-9 level did not change after 5-ALA treatment. However, the expression of caspase-9 in RGK cells was higher than RGM normal cells when 5-ALA was not treated. This may be because the production level of ROS is higher and many signal transductions are activated in cancer cells. Meanwhile, the expression level of cleaved caspase- 9 increased slightly in RGM at $24 \mathrm{~h}$ treatment (Supplemental Fig. 2*). Figure 4B shows that 5-ALA treatment increases the expression of phosphorylated p53 protein in RGM cells. However, the expression tended to decrease after 5-ALA treatment in RGK cells. In addition, phosphorylated p53 expression increased in RGM treated for $24 \mathrm{~h}$ (Supplemental Fig. $2 *$ ). These results indicate that oxidative stress-dependent apoptosis is induced by 5-ALA in RGM cells.

ROS measurement in living cells. We examined ROS production in living gastric normal and cancer cells after 5-ALA treatment by ESR. ESR is a device which detects ROS easily with high-sensitivity. Figure 5 shows weak peaks were observed in RGM control cells. Treatment with $50 \mu \mathrm{M}$ 5-ALA enhanced signal intensity. Compared to RGM cells, signal peaks in RGK cells were a little stronger in the control, and they were almost the same intensity after 5-ALA treatment. In general, ROS production level in cancer cells is higher than in normal cells because cancer cellular mitochondria have mutation and electron leakage occurs in electron transport chain. These results indicate that gastric normal cells are more easily damaged and produce ROS in greater levels than cancer cells when treated with 5-ALA although RGK cells generated ROS more than RGM cells without treatment. In addition, generated ROS were likely to hydroxyl radical because of characteristical four signal peaks.

Cell viability measurement after co-treatment with NAC and 5-ALA. 5-ALA treatment of gastric normal cells induced apoptosis and ROS generation. To examine the relationship between 5-ALA treatment, ROS production, and cell death in detail, an antioxidant NAC was used to co-treat with 5-ALA, measuring cell viability. The $50 \mu \mathrm{M} 5$-ALA caused cell damage and viability decreased (Fig. 6). NAC treatment recovered the viability in a dose-dependent manner up to $50 \mu \mathrm{M}$, and over $50 \mu \mathrm{M}$ of NAC induced cytotoxicity followed by decrease of viability again in a dose-dependent. These results indicate that ROS induced by 5-ALA treatment damaged cells but scavenging the ROS with NAC suppressed cellular apoptosis in a gastric normal cell line.

p53 inhibitor treatment and cell viability. In order to investigate the role of p53 in 5-ALA-induced apoptosis, we examined cell viability of RGM after co-treatment with an inhibitor of p53-dependent apoptosis and transcription and 5ALA. Figure 7 shows that only $50 \mu \mathrm{M}$ 5-ALA treatment decreased cell viability and the decrease in cell viability caused by 5-ALA recovered in a dose-dependent manner with p53 inhibitor up to $5 \mu \mathrm{M}$. Greater than $5 \mu \mathrm{M}$ inhibitor caused dose-dependent cellular injury by the toxicity of the reagent itself. Although 5-ALA itself showed cytotoxicity in this study, cell viability was increased by a p53-dependent apoptosis inhibitor. These results indicate that apoptotic cell death of RGM caused by 5-ALA was dependent on the $\mathrm{p} 53$ signalling process. Therefore, inhibition of $\mathrm{p} 53$ was likely to induce the recovery of viability.

\section{Discussion}

In this study, we investigated the effect of 5-aminolevulinic acid on oxidative stress in normal and cancerous gastric cells and elucidated the involved cellular apoptotic pathway. 5-ALA is a precursor of heme and is thought to be an important nutrient for living organisms including plants. However, a certain concentration of 5-ALA reduced normal cellular viability whereas cancer cells proliferated in the same condition (Fig. 1 and Supplemental Fig. ${ }^{*}$ ). 5-ALA was also shown to be an oxidative stress inducer in normal cells (Fig. 5). On the other hand, 5-ALA has a role to promote cell growth, as described above. Therefore, low concentration of 5-ALA may gradually increase the cell viability, especially in cancer cell line.

ROS have a bifunctional property dependent on their concentration; they function as a signalling mediator at low concentrations and cause protein dysfunction or cell death at higher concentrations. ${ }^{(35)}$ In general, ROS production is enhanced in cancer cells, 
A RGM
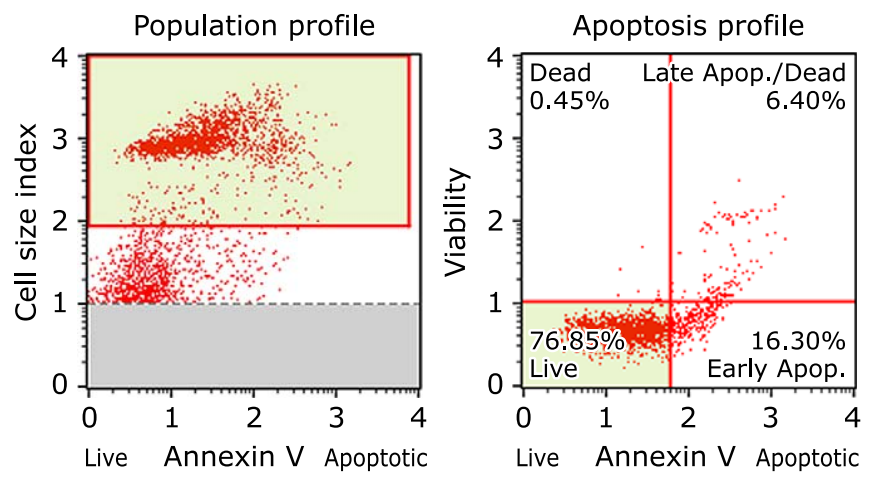

\section{RGK}
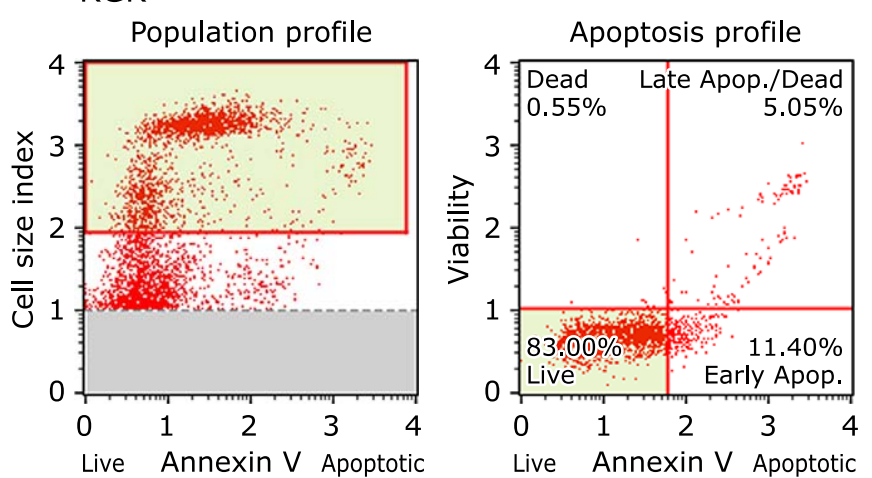
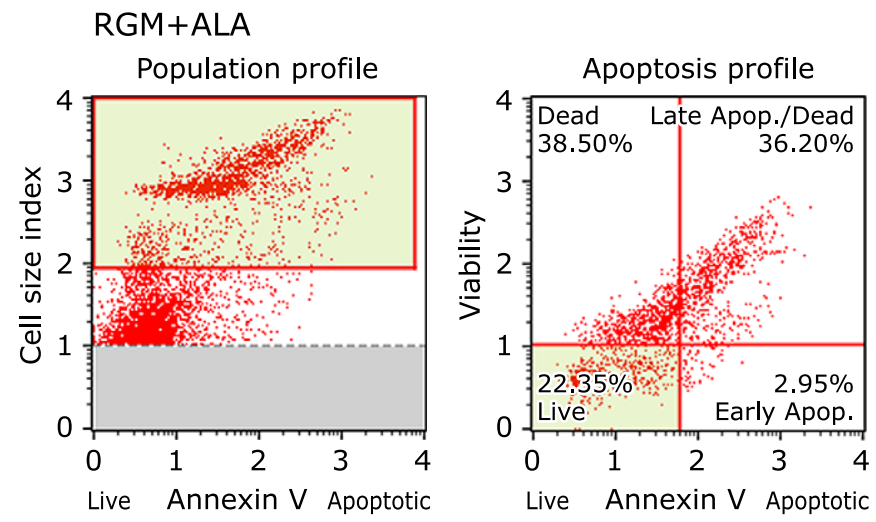

\section{RGK+ALA}
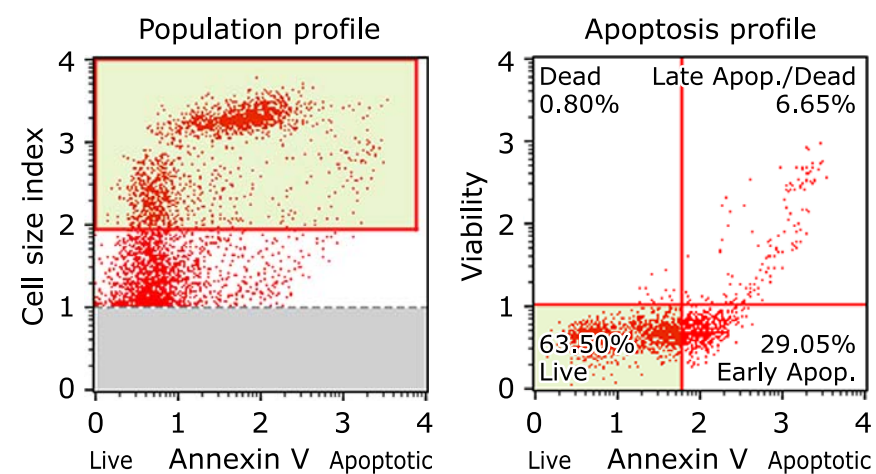

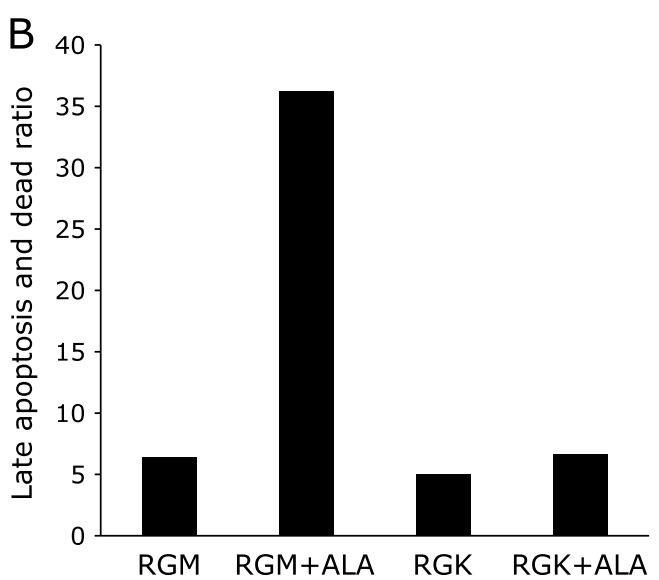

Fig. 2. Annexin $V$ apoptosis assay to estimate the ratio of cellular apoptosis in RGM and RGK cells using a Muse Annexin $V$ \& Dead Cell Kit after treatment with $50 \mu \mathrm{M}$ 5-ALA for $48 \mathrm{~h}$. Figure $2 \mathrm{~A}$ shows the histogram of cellular state and Fig. $2 \mathrm{~B}$ is the bar graph data of late apoptosis and dead cell ratio. Cellular apoptosis and dead cell ratio of RGM cells increased remarkably with 5-ALA treatment, whereas only a small increase of early apoptotic RGK cells was observed.

and excess amounts of ROS mediate various signal transductions and the activation of various proteins. ${ }^{(36)}$ In addition, cancer cells develop protective systems against ROS such as superoxide dismutase and glutathione. These antioxidative systems enable cancer cells to survive with high levels of ROS. Such high concentrations of ROS cause harmful effects to normal cells, and can result in apoptosis. Namely, it is inferred that resistance for ROS in cancer cell is greater than in normal cell. Therefore, RGK cells would be dead in higher concentration of 5-ALA than in RGM cells. In fact, RGK cells were dead at $100 \mu \mathrm{M}$ and higher ROS generation was confirmed (Supplemental Fig. $3 *$ ). However, the cellular damage was not so serious and the viability was recovered with $1 \mu \mathrm{M}$ of NAC, and caspase-9 expression level was not changed (Supplemental Fig. $4 *$ and $5 *$ ). In addition, we confirmed elevated production of ROS, positive annexin $\mathrm{V}$ detection and enhanced expression of caspase-3/7 in gastric normal cells, but not in cancer cells, after treatment with $50 \mu \mathrm{M}$ of 5-ALA (Fig. 2, 3 and 5). These results show that an increase in ROS generation caused by 5-ALA induces apoptotic cell death in normal cells.

Apoptosis is induced by various complicated signalling pathways associated with Fas ligand (FasL), p53 protein, and endoplasmic reticulum stress. ${ }^{(37-39)} \mathrm{Lu}$ et al. ${ }^{(40)}$ reported that FasL, a member of tumour necrosis factor super family, induced apoptosis of macrophage RAW 264.7 cells, and that inhibition of transcrip- 
A RGM
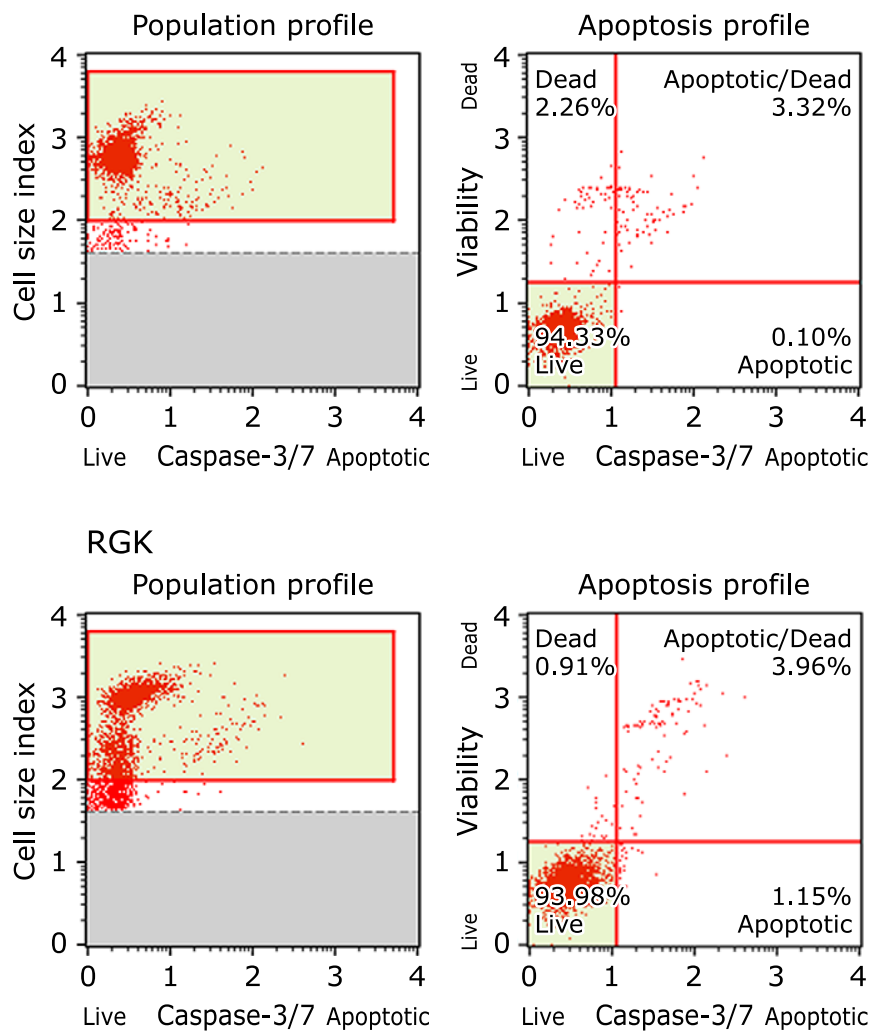

$\mathrm{RGM}+\mathrm{ALA}$
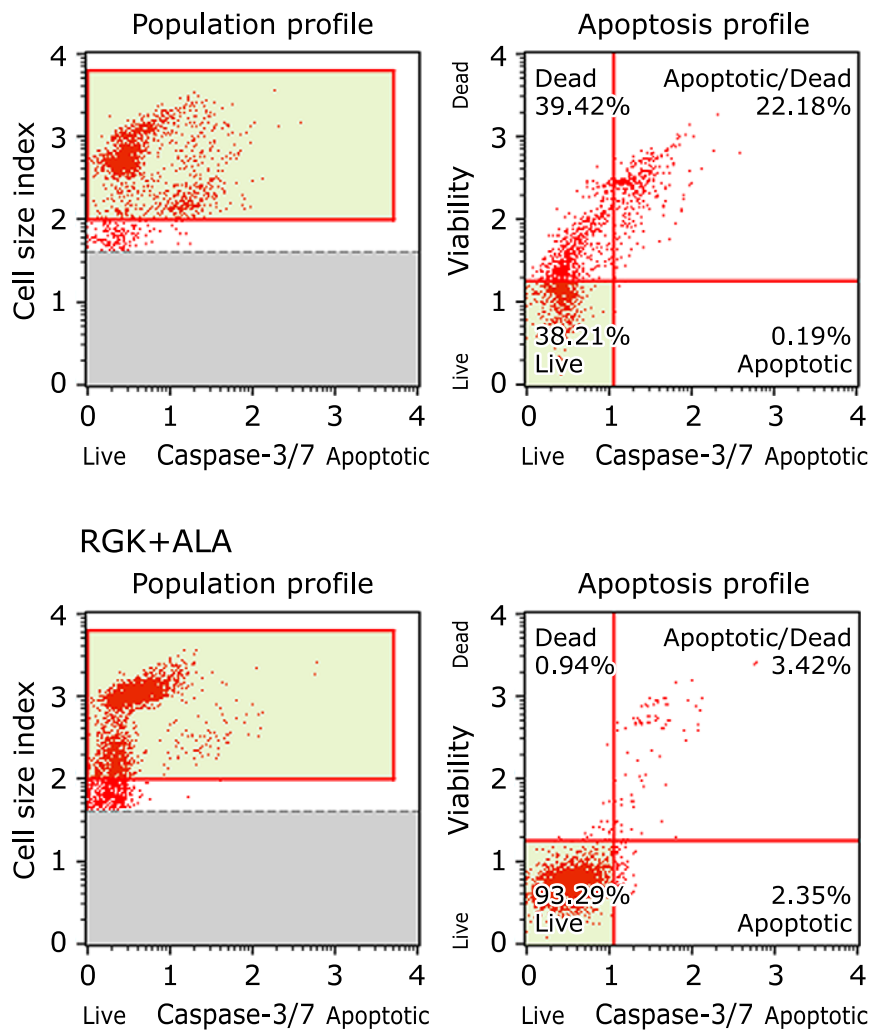

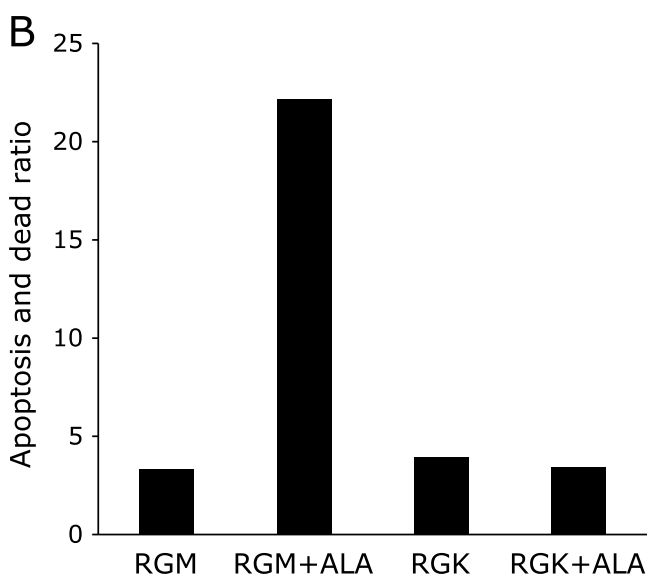

Fig. 3. Cellular apoptotic ratio of RGM and RGK cells treated with $50 \mu \mathrm{M} 5$-ALA for $48 \mathrm{~h}$ or untreated, estimated by detection of caspase-3/7 positive cells. Figure 3A shows the histogram of cellular state and Fig. 3B is the bar graph data of apoptosis and dead cell ratio. 5-ALA enhanced the ratio of apoptotic and dead cells in the RGM cell line. The ratio in RGK was unchanged by 5-ALA treatment.

tion factor NF- $\kappa \mathrm{B}$ enhanced FasL-induced apoptosis. NF- $\kappa \mathrm{B}$ is activated by oxidative stress and is constitutively activated in many types of cancer. ${ }^{(41,42)}$ In addition, NF- $\kappa B$ promotes the proliferation of cancer cells and helps them escape cell death. ${ }^{(43)}$ Thus, severe cancer cell death would not be induced in our study. Oxidative stress induced by 5-ALA in normal cells may also activate NF- $\kappa B$, meaning induction of apoptosis would not occur through FasL signalling.

p53 protein, often called a guardian of the genome, plays an important role in the maintenance of cellular quality and responds to DNA damage, abnormal oncogenic events, hypoxia etc., inducing apoptosis when appropriate. ${ }^{(44)}$ p53 is also activated by oxidative stress, promoting release of cytochrome $c$ from mito- chondria, followed by induction of apoptosis via activation of caspase-9. ${ }^{(34,45)}$ We showed that administration of 5-ALA induced activation of p53 and caspase-9 in normal cells (Fig. 4). Furthermore, addition of an antioxidant NAC suppressed the decrease in cell viability and an inhibitor of p53-dependent apoptosis produced resistance to 5-ALA-induced cell death in normal cells (Fig. 6 and 7). Therefore, we hypothesize that ROS generated by 5-ALA activate caspase-9 via p53 activation, subsequently inducing apoptosis in normal cells. Meanwhile, 5-ALA is reported to oxidize a constituent of the mitochondrial inner membrane, cardiolipin, which damages mitochondria. ${ }^{(12)}$ Macip et al. ${ }^{(46)}$ reported that induction of p53 is associated with accumulation of ROS in mitochondria and influences the decision for apoptosis. 
A

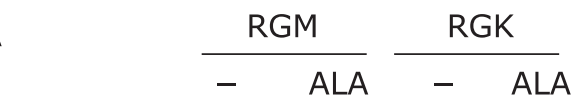

Full length

caspase-9

Cleaved

caspase-9

$\beta$-actin
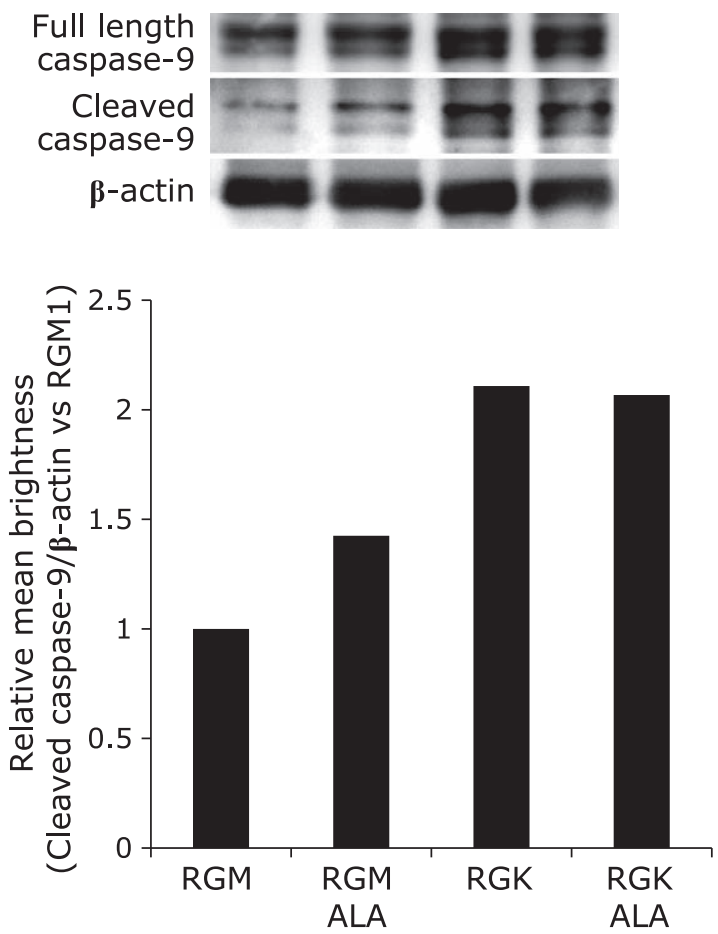

B
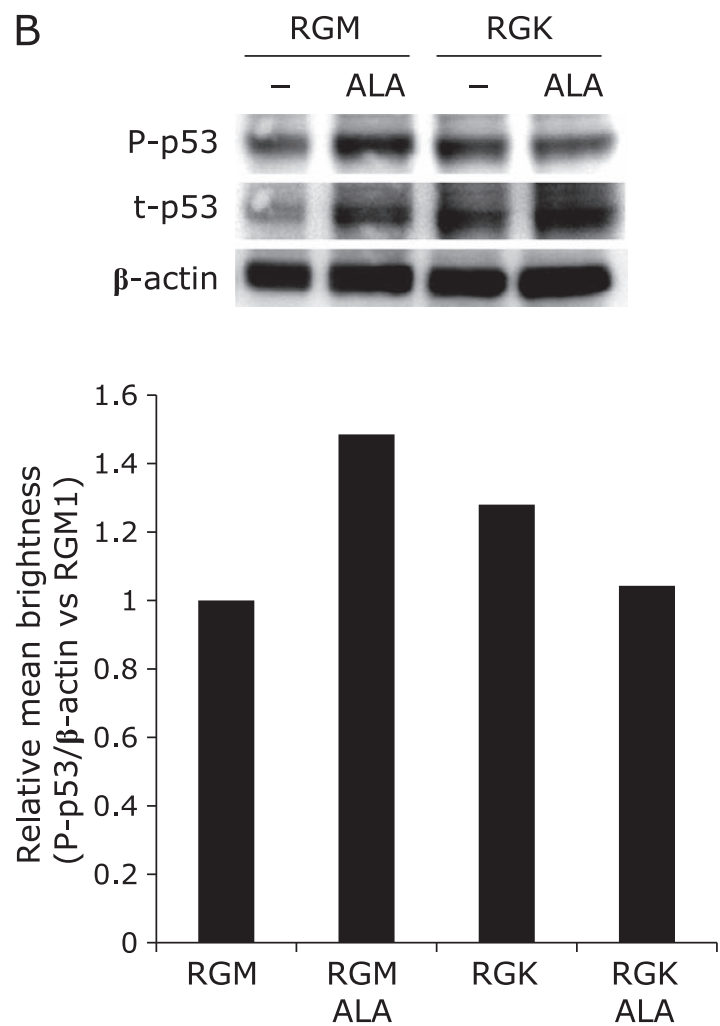

Fig. 4. Protein expression transitions after treatment with $50 \mu \mathrm{M} 5$-ALA for $48 \mathrm{~h}$ analysed by western blotting. (A) The expression level of cleaved caspase-9. 5-ALA enhanced expression in RGM cells. While the basal level was higher in RGK cells, 5-ALA did not markedly alter the level. (B) The level of phosphorylated p53 protein. Elevated level of phosphorylated p53 was observed in RGM cells after 5-ALA exposure. Expression was prone to decrease in RGK cells after 5-ALA treatment.
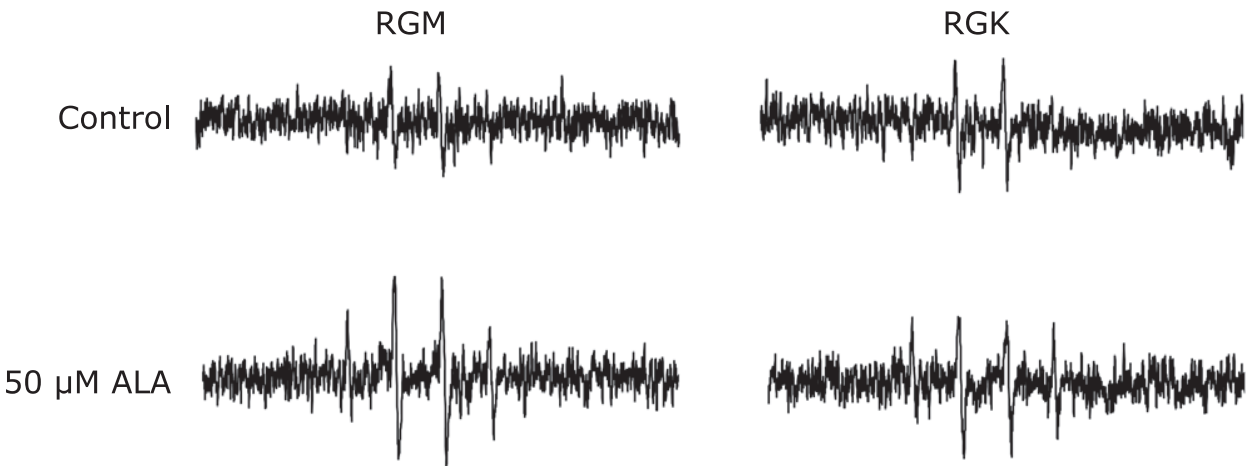

Fig. 5. Detection of ROS in living cells by electron spin resonance (ESR). ROS generation levels are represented as ESR signal intensities. Cellular ROS production in RGM cells increased but did not increase in RGK cells after incubation with $50 \mu \mathrm{M} 5$-ALA for $48 \mathrm{~h}$.

Accordingly, 5-ALA may enhance mitochondrial ROS generation and induce apoptosis. On the other hand, Schuler et al. ${ }^{(47)}$ reported that a caspase inhibitor suppressed cell death in p53 cDNAtransduced cells, whereas NAC did not. In this study, we suggest that neutralization of 5-ALA-induced intracellular ROS by NAC prevented activation of $\mathrm{p} 53$, resulting in suppression of cell death. p53 gene mutations have been reported in many types of cancers, and expression of mutant p53 grants cells the ability to evade apoptosis. ${ }^{(48)}$ Consequently, apoptosis in RGK cancer cells was not observed because p53 may have mutated.

In conclusion, 5-aminolevulinic acid promotes generation of
ROS and induction of apoptosis via activation of $\mathrm{p} 53$ and caspases in gastric normal cells but increases viability in gastric cancer cells. However, 5-ALA has already been utilized as a prodrug for PDT to treat cancer in clinical site. Due to its cytotoxic effect on normal cells, long-term dosing may be harmful to patients. As mentioned above, 5-ALA is reported to be excreted from tissue and body in $48 \mathrm{~h}$. However, we showed the role of 5-ALA as an oxidative stressor in this study and we also have reported that 5-ALA has a tendency to accumulate in cancer cells. ${ }^{(31)}$ Therefore, 5-ALA may have a risk to damage normal cells and reinforce cancer cells whereas PDT is a superior cancer treatment. 


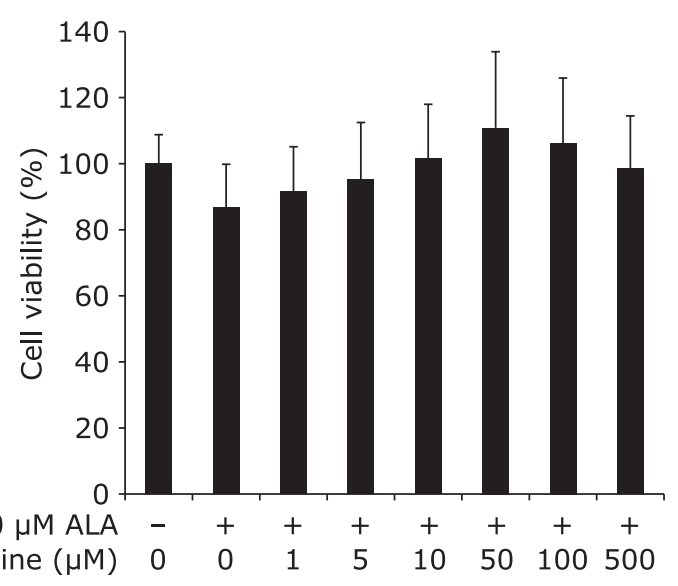

Fig. 6. $N$-acetyl-L-cysteine (NAC) was applied with $50 \mu \mathrm{M}$ 5-ALA measuring viability of RGM cells. The decrease in viability caused by 5-ALA was ameliorated by NAC in a dose dependent manner up to $50 \mu$ M. $n=6$, Error bar; SD.

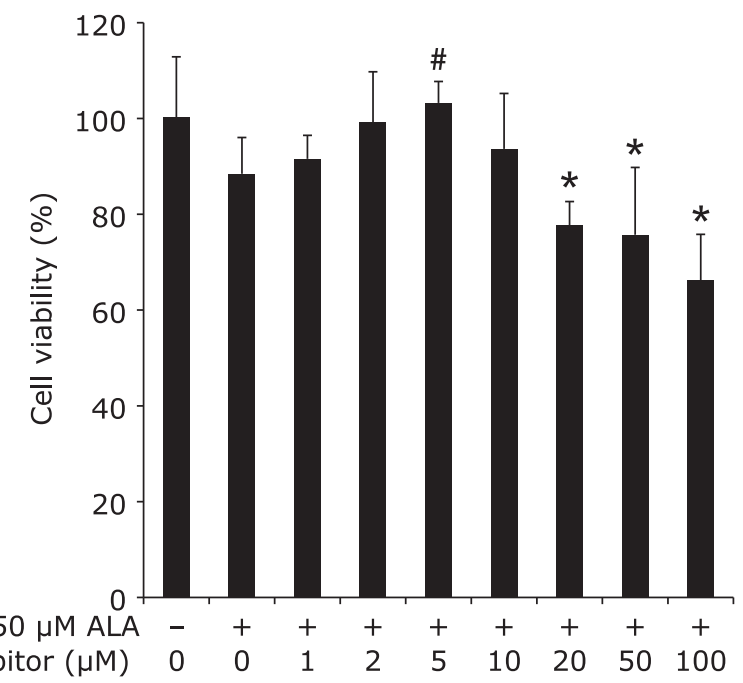

Fig. 7. RGM cell viability after treatment with $p 53-$ dependent apoptosis and transcription inhibitor with $50 \mu \mathrm{M}$ 5-ALA. p53 inhibitor improved the viability in a dose dependent manner up to $5 \mu \mathrm{M}$. Statistical significance was tested by Tukey's test. $n=6$, Error bar; SD. ${ }^{*} p<0.01$ vs \#.

\section{Acknowledgments}

The authors gratefully thank Kenichi Iwasaki, Ken Nakayama and Nobuhiro Ohkohchi, who belong to the Department of Gastroenterological and Hepatobiliary Surgery and Organ Transplantation, Faculty of Medicine, University of Tsukuba for use of the MUSE Cell Analyzer. They also thank Aki Hirayama, who belongs to Center for Integrative Medicine, Tsukuba University of Technology for use of the ESR system. This study was partially supported by JSPS KAKENHI Grant Number JP17K15007.

\section{Conflict of Interest}

No potential conflicts of interest were disclosed.

\section{References}

1 Morokuma Y, Yamazaki M, Maeda T, et al. Hair growth stimulatory effect by a combination of 5-aminolevulinic acid and iron ion. Int J Dermatol 2008; 47: 1298-1303.

2 Sato K, Matsushita K, Takahashi K, et al. Dietary supplementation with 5aminolevulinic acid modulates growth performance and inflammatory responses in broiler chickens. Poult Sci 2012; 91: 1582-1589.

3 Chen YJ, Kim IH, Cho JH, Min BJ, Yoo JS, Wang Q. Effect of $\delta$-aminolevulinic acid on growth performance, nutrient digestibility, blood parameters and the immune response of weanling pigs challenged with Escherichia coli lipopolysaccharide. Livest Sci 2008; 114: 108-116.

4 Robey RW, Steadman K, Polgar O, Bates SE. ABCG2-mediated transport of photosensitizers: potential impact on photodynamic therapy. Cancer Biol Ther 2005; 4: 187-194.

5 Marbacher S, Klinger E, Schwyzer L, et al. Use of fluorescence to guide resection or biopsy of primary brain tumors and brain metastases. Neurosurg Focus 2014; 36: E10.

6 Hotta Y, Tanaka T, Takaoka H, Takeuchi Y, Konnai M. Promotive effects of 5-aminolevulinic acid on the yield of several crops. Plant Growth Regul 1997; 22: 109-114.

7 Watanabe K, Tanaka T, Hotta , Y, Kuramochi H, Takeuchi Y. Improving salt tolerance of cotton seedlings with 5-aminolevulinic acid. Plant Growth Regul 2000; 32: 97-101.

8 Hotta Y, Tanaka T, Bingshan L, Takeuchi Y, Konnai M. Improvement of cold resistance in rice seedlings by 5 -aminolevulinic acid. J Pestic Sci 1998; 23: 29-33.

9 Rebeiz CA, Montazer-Zouhoor A, Hopen HJ, Wu SM. Photodynamic herbicides: 1. Concept and phenomenology. Enzyme Microb Tech 1984; 6: 390 396.

10 Ahamed M, Siddiqui MK. Low level lead exposure and oxidative stress: current opinions. Clin Chim Acta 2007; 383: 57-64.

11 Ito S, Miyoshi N, Degraff WG, Nagashima K, Kirschenbaum LJ, Riesz P. Enhancement of 5-aminolevulinic acid-induced oxidative stress on two cancer cell lines by gold nanoparticles. Free Radic Res $2009 ;$ 43: 1214-1224.

12 Oteiza PI, Bechara EJ. 5-Aminolevulinic acid induces lipid peroxidation in cardiolipin-rich liposomes. Arch Biochem Biophys 1993; 305: 282-287.

13 Schieber M, Chandel NS. ROS function in redox signaling and oxidative stress. Curr Biol 2014; 24: R453-R462.

14 Ray PD, Huang BW, Tsuji Y. Reactive oxygen species (ROS) homeostasis and redox regulation in cellular signaling. Cell Signal 2012; 24: 981-990.

15 Klaunig JE, Kamendulis LM, Hocevar BA. Oxidative stress and oxidative damage in carcinogenesis. Toxicol Pathol 2010; 38: 96-109.

16 Piechota-Polanczyk A, Fichna J. Review article: the role of oxidative stress in pathogenesis and treatment of inflammatory bowel diseases. Naunyn Schmiedebergs Arch Pharmacol 2014; 387: 605-620.

17 Bedard K, Krause KH. The NOX family of ROS-generating NADPH oxidases: physiology and pathophysiology. Physiol Rev 2007; 87: 245-313.

18 Cao SS, Kaufman RJ. Endoplasmic reticulum stress and oxidative stress in cell fate decision and human disease. Antioxid Redox Signal 2014; 21: 396413.

19 Marchi S, Giorgi C, Suski JM, et al. Mitochondria-ros crosstalk in the control of cell death and aging. J Signal Transduct 2012; 2012: 329635.

20 Bratic I, Trifunovic A. Mitochondrial energy metabolism and ageing. Biochim Biophys Acta 2010; 1797: 961-967.

21 van Gisbergen MW, Voets AM, Starmans MH, et al. How do changes in the mtDNA and mitochondrial dysfunction influence cancer and cancer therapy? Challenges, opportunities and models. Mutat Res Rev Mutat Res 2015; 764 $16-30$.

22 Sullivan LB, Chandel NS. Mitochondrial reactive oxygen species and cancer. Cancer Metab 2014; 2: 17.

23 Chatterjee A, Mambo E, Sidransky D. Mitochondrial DNA mutations in human cancer. Oncogene 2006; 25: 4663-4674.

24 Chen EI. Mitochondrial dysfunction and cancer metastasis. J Bioenerg Biomembr 2015; 44: 619-622.

25 Tamura M, Matsui H, Tomita T, et al. Mitochondrial reactive oxygen species accelerate gastric cancer cell invasion. J Clin Biochem Nutr 2014; 54: 12-17.

26 Panieri E, Santoro MM. ROS homeostasis and metabolism: a dangerous liason in cancer cells. Cell Death Dis 2016; 7: e2253.

27 Kobayashi I, Kawano S, Tsuji S, et al. RGM1, a cell line derived from normal gastric mucosa of rat. In Vitro Cell Dev Biol Anim 1996; 32: 259-261. 
28 Shimokawa O, Matsui H, Nagano Y, et al. Neoplastic transformation and induction of $\mathrm{H}^{+}, \mathrm{K}^{+}$-adenosine triphosphatase by $N$-methyl- $N^{\prime}$-nitro- $N$-nitrosoguanidine in the gastric epithelial RGM-1 cell line. In Vitro Cell Dev Biol Anim 2008; 44: 26-30.

29 Chen HM, Yu CH, Lin HP, Cheng SJ, Chiang CP. 5-Aminolevulinic acidmediated photodynamic therapy for oral cancers and precancers. J Dent Sci 2012; 7: 307-315.

30 Elmore S. Apoptosis: a review of programmed cell death. Toxicol Pathol 2007; 35: 495-516.

31 Ito H, Tamura M, Matsui H, Majima HJ, Indo HP, Hyodo I. Reactive oxygen species involved cancer cellular specific 5-aminolevulinic acid uptake in gastric epithelial cells. J Clin Biochem Nutr 2014; 54: 81-85.

32 Zuo Y, Xiang B, Yang J, et al. Oxidative modification of caspase-9 facilitates its activation via disulfide-mediated interaction with Apaf-1. Cell Res 2009; 19: 449-457.

33 Andoh T, Chock PB, Chiueh CC. The roles of thioredoxin in protection against oxidative stress-induced apoptosis in SH-SY5Y Cells. J Biol Chem 2002; 277: 9655-9660.

34 Liu D, Xu Y. p53, oxidative stress, and aging. Antioxid Redox Signal 2011; 15: 1669-1678.

35 Circu ML, Aw TY. Reactive oxygen species, cellular redox systems and apoptosis. Free Radic Biol Med 2010; 48: 749-762.

36 Liou GY, Storz P. Reactive oxygen species in cancer. Free Radic Res 2010; 44. DOI: $10.3109 / 10715761003667554$

37 Waring P, Müllbacher A. Cell death induced by the Fas/Fas ligand pathway and its role in pathology. Immunol Cell Biol 1999; 77: 312-317.

38 Haupt S, Berger M, Goldberg Z, Haupt Y. Apoptosis - the p53 network. $J$
Cell Sci 2003; 116: 4077-4085.

39 Szegezdi E, Logue SE, Gorman AM, Samali A. Mediators of endoplasmic reticulum stress-induced apoptosis. EMBO Rep 2006; 7: 880-885.

40 Lu B, Wang L, Medan D, et al. Regulation of Fas (CD95)-induced apoptosis by nuclear factor- $\kappa \mathrm{B}$ and tumor necrosis factor- $\alpha$ in macrophages. $\mathrm{Am} \mathrm{J}$ Physiol Cell Physiol 2002; 283: C831-C838.

41 van den Berg R, Haenen GR, van den Berg H, Bast A. Transcription factor $\mathrm{NF}-\kappa \mathrm{B}$ as a potential biomarker for oxidative stress. Brit J Nutr 2001; 86 Suppl 1: S121-S127.

42 Hoesel B, Schmid JA. The complexity of NF- $\kappa$ B signaling in inflammation and cancer. Mol Cancer 2013; 12: 86.

43 Hassanzadeh P. Colorectal cancer and NF-кB signaling pathway. Gastroenterol Hepatol Bed Bench 2011; 4: 127-132.

44 Dai C, Gu W. p53 post-translational modification: deregulated in tumorigenesis. Trends Mol Med 2010; 16: 528-536.

45 Wu GS, Ding Z. Caspase 9 is required for p53-dependent apoptosis and chemosensitivity in a human ovarian cancer cell line. Oncogene 2002; 21: 1-8.

46 Macip S, Igarashi M, Berggren P, Yu J, Lee SW, Aaronson SA. Influence of induced reactive oxygen species in p53-mediated cell fate decisions. Mol Cell Biol 2003; 23: 8576-8585.

47 Schuler M, Bossy-Wetzel EB, Goldstein JC, Fitzgerald P, Green DR. p53 induces apoptosis by caspase activation through mitochondrial cytochrome $c$ release. J Biol Chem 2000; 275: 7337-7342.

48 Rivlin N, Brosh R, Oren M, Rotter V. Mutations in the p53 tumor suppressor gene: important milestones at the various steps of tumorigenesis. Genes Cancer 2011; 2: 466-474. 